\title{
International Journal of

\section{Does EuroSCORE Predict intensity of Postoperative Care and Rehabilitation Parameters? A Prospective Evaluation}

Volkhard Goeber ${ }^{1}$, Uta Faeh ${ }^{1}$, Dorothee Keller ${ }^{1}$, Hugo Saner ${ }^{2}$, Thierry P. Carrel ${ }^{1}$ and Lars Englberger ${ }^{1}$

${ }^{1}$ Department of Cardiovascular Surgery, University Hospital Bern and University of Bern, Switzerland

${ }^{2}$ Cardiovascular Prevention and Rehabilitation, University Hospital Bern and University of Bern, Switzerland

"Corresponding author: Volkhard Goeber, Department of Cardiovascular Surgery, University Hospital Bern, CH-3010 Bern, Switzerland, Tel: +41 31 632 23 87; Fax: +4131632 44 43; E-mail: volkhard.goeber@insel.ch

Received date: 21 March 2014; Accepted date: 26 April 2014; Published date: 28 April 2014

Copyright: (c) 2014 Goeber V, et al. This is an open-access article distributed under the terms of the Creative Commons Attribution License, which permits unrestricted use, distribution, and reproduction in any medium, provided the original author and source are credited.

\begin{abstract}
Objective: An increasing number of elderly and multi-morbid patients undergo cardiac surgery which results in increased postoperative morbidity, prolonged Intensive Care Unit (ICU) length of stay (LOS) and higher hospital costs. We aimed to evaluate the model of the European System for Cardiac Operative Risk Evaluation (EuroSCORE) for the prediction of ICU LOS, hospital LOS, required daily nursing effort, and the type of cardiac rehabilitation.
\end{abstract}

Methods: Prospective observational evaluation of 505 consecutive adult patients (mean age $65.1 \pm 12.1$ years, $25.7 \%$ female) who underwent cardiac and/or thoracic aortic thoracic surgery with Cardiopulmonary Bypass (CPB).

Results: Median additive and logistic EuroSCORE was 5 (Interquartile Range (IQR) 3-7) and 5.8 (IQR 2.6-14.1), respectively. In univariate analysis both additive and logistic EuroSCORE were significantly associated with prolonged ICU LOS, prolonged hospital LOS, higher daily nursing effort, and the type of cardiac rehabilitation (inpatient versus outpatient), $p<0.001$ for all correlations.

Multivariate analysis including other clinically relevant variables (CPB duration, type of operation, age, Body Mass Index (BMI), urgency of surgery, Left Ventricular Ejection Fraction (LVEF)) showed higher additive EuroSCORE and higher logistic EuroSCORE, independently associated with prolonged ICU LOS, prolonged hospital LOS, and higher daily nursing effort. However, EuroSCORE did not independently predict the type of rehabilitation.

Conclusion: The EuroSCORE model can be used to identify patients with elevated risk for prolonged ICU LOS, prolonged hospital LOS and higher intensity of postoperative workload, and it is predictive for the type of rehabilitation. This conclusion may contribute to optimize the management of hospital bed capacity as well as a systematic planning of ICU resources, postoperative nursing care and cardiac rehabilitation.

Keywords: EuroSCORE, Cardiac surgery; Rehabilitation, Workload of nursing care

\begin{abstract}
Abbreviations:
BMI: Body Mass Index; CABG: Coronary Artery Bypass Graft; CPB: Cardiopulmonary Bypass; EuroSCORE: European System for Cardiac Operative Risk Evaluation; IABP: Intra-aortic Balloon Pump; ICU: Intensive Care Unit; IMC: Intermediate Care Unit; LVEF: Left Ventricular Ejection Fraction; MI: Myocardial Infarction; PRN: Projet de Recherche en Nursing; SD: Standard Deviation
\end{abstract}

\section{Introduction}

Patient's profile in cardiac surgery has considerably changed over the last 25 years [1]. Despite the increasing number of elderly and multi-morbid patients, in-hospital mortality in cardiac surgery remained unchanged or could be reduced [1]. In consequence, a higher rate of postoperative morbidity is associated with prolonged ICU LOS and, in this clinical setting, with a remarkable increase of individual health care costs per patient [2-4]. Therefore, optimal utilization of a hospital's resources becomes more and more important.

Prediction of ICU LOS and hospital LOS are decisive information for a hospital's management to allocate resources and to estimate hospital costs. In addition, estimation of intensity of postoperative nursing care during hospitalization allows calculation of required human resources for adequate nursing in a specified patient population. Patients who have undergone cardiac surgery routinely participate during hospitalization (Phase I rehabilitation) and after discharge (Phase II rehabilitation) in cardiac rehabilitation programs which are performed as inpatient or outpatient programs. Preoperative prediction of the appropriate type of rehabilitation is desirable for an optimal planning of the entire therapeutic concept.

The EuroSCORE model was first introduced in 1999 as an additive predictive system for 30 -day mortality after cardiac surgery $[5,6]$. Several external validation studies justified the widespread use of both of the additive EuroSCORE and the logistic EuroSCORE, introduced in 2003 [7-9]. In addition, it has been demonstrated that EuroSCORE has also predictive validity for other outcome measures than operative mortality [9]. Recently, the modified EuroSCORE II has been 
Citation: Goeber V, Faeh U, Keller D, Saner H, Carrel TP, et al. (2014) Does EuroSCORE Predict intensity of Postoperative Care and Rehabilitation Parameters? A Prospective Evaluation. Int J Phys Med Rehabil S5: 007. doi:10.4172/2329-9096.S5-007

Page 2 of 5

introduced as a further advancement of additive and logistic EuroSCORE [10].

The aim of this prospective observational cohort study was to evaluate the additive and logistic EuroSCORE model for the prediction of ICU LOS, hospital LOS, and required postoperative nursing effort in patients undergoing cardiac surgery. In addition, we investigated if EuroSCORE may predict the type of cardiac rehabilitation.

\section{Methods}

\section{Study population}

In this prospective, single-center (University Hospital Bern, Switzerland) observational study, 505 consecutive adult patients were included who had undergone cardiac and/or thoracic aortic surgery with cardiopulmonary bypass $(\mathrm{CPB})$ within a six month period. The initial intent of this study was to evaluate intensity of nursing care after cardiac surgery, specifically focused on nursing effort and type of cardiac rehabilitation. The study was supported by the Department of Health and Social Welfare of the Canton of Bern and was approved by the ethical review committee. All patients provided written informed consent.

Preoperative risk factors were recorded according to the EuroSCORE model $[6,11]$. For study purpose prolonged ICU LOS was defined as $>2$ subsequent nights on ICU, and prolonged hospital LOS was defined as $>/=8$ days of postoperative hospitalization.

To determine daily nursing effort after transfer from ICU to the regular ward, the standardized "Projet de Recherche en Nursing" (PRN) system was used [12]. The PRN system, developed for evaluation of nursing personnel requirements, had been extensively validated [13-15], and had been used in clinical routine in our hospital for more than a decade. Briefly, for standardized recording of nursing workload, 214 indicators or tasks are considered for cumulative description of patient-specific measures within a twenty-four hour period. A point value is assigned to each indicator, whereby each point represents five minutes of nursing work. This "average" time methodology is based on the average amount of time required for the measuring on an average day, by an average nurse, for an average patient [16].

For all patients after cardiac and/or thoracic aortic operations, participation in a cardiac rehabilitation was intended. Rehabilitation was performed either as inpatient or outpatient program. Inpatient rehabilitation was preferred for patients with a complicated postoperative course, distinct co-morbidities, the need for intensive medical care, the need for milieu change or a lack of homecare. Benefits of outpatient rehabilitation include the patient's possibility of part-time work, an extended time period of rehabilitation, the closeness to the patient's home, and the inclusion of relatives.

Definitions of the Society of Thoracic Surgeons Adult Database were used for data recording [www.sts.org/national-database] [17]. In addition, preoperative renal failure was defined as serum creatinine > $200 \mu \mathrm{mol} / \mathrm{l}$ and/or dialysis, postoperative renal failure as elevation of serum creatinine $>172 \mu \mathrm{mol} / \mathrm{l}$ and elevation $>50 \%$ compared to preoperative baseline creatinine. Urgent operation was defined as $<24$ hours after diagnosis/indication [17].

\section{Data analysis}

In the descriptive statistics, metric variables are presented as mean and standard deviation, categories are presented as number and percentage. To investigate the relationship between PRN and EuroSCORE (additive as well as logistic), we calculated a correlation analysis (Pearson). To adjust for suspected confounders, we performed multiple regression analyses: a linear regression with mean PRN as dependent variable and several logistic regression analyses with prolonged hospital LOS, prolonged ICU LOS and type of rehabilitation (inpatient or outpatient) as dependent variables, respectively. As suspected confounders, we included $\mathrm{CPB}$ duration, type of cardiac operation (isolated Coronary Artery Bypass Graft (CABG) or other cardiac surgery), age, BMI, urgency of surgery (elective or urgent), and preoperative LVEF. Alternatively, we performed a separate analysis for additive and logistic EuroSCORE. All p-values and confidence intervals are 2 -sided, a $\mathrm{p}<0.05$ is considered significant.

All statistical analyses were performed using the computerized software package SPSS for Windows Version 17.0.

\section{Results}

Mean age in the entire study population was $65.1 \pm 12.1$ years, 130 (25.7\%) patients were female. Complete patient demographics and perioperative data are presented in the Table. Median additive EuroSCORE was 5 (IQR 3 - 7), and median logistic EuroSCORE was 5.8 (IQR 2.6 - 14.1).

\begin{tabular}{|c|c|}
\hline & $(n=505)$ \\
\hline Age, years & $65.1 \pm 12.1$ \\
\hline Female sex, $\mathrm{n}(\%)$ & $130(25.7)$ \\
\hline \multicolumn{2}{|l|}{ Cardiovascular risk factors } \\
\hline Diabetes mellitus, $\mathrm{n}(\%)$ & $129(25.5)$ \\
\hline Hypertension, n (\%) & $380(75.4)$ \\
\hline Adiposity, n (\%) & $229(45.3)$ \\
\hline Dyslipidemia, n (\%) & $300(59.5)$ \\
\hline Smoker, n (\%) & $245(48.6)$ \\
\hline Body Mass Index, $\mathrm{kg} / \mathrm{m}^{2}$ & $26.9 \pm 4.6$ \\
\hline Family history, n (\%) & $121(24.0)$ \\
\hline \multicolumn{2}{|l|}{ Left ventricular ejection fraction } \\
\hline$\geq 50, \mathrm{n}(\%)$ & $375(74.3)$ \\
\hline $30-49, \mathrm{n}(\%)$ & 99 (19.6) \\
\hline$\leq 29, \mathrm{n}(\%)$ & $31(6.1)$ \\
\hline \multicolumn{2}{|l|}{ EuroSCORE } \\
\hline Additive, median [IQR] & $5[3-7]$ \\
\hline Logistic, median [IQR] & $5.8[2.6-14.1]$ \\
\hline Peripheral artery disease, $\mathrm{n}(\%)$ & $73(14.5)$ \\
\hline Chronic lung disease, $\mathrm{n}(\%)$ & $77(15.4)$ \\
\hline
\end{tabular}


Citation: Goeber V, Faeh U, Keller D, Saner H, Carrel TP, et al. (2014) Does EuroSCORE Predict intensity of Postoperative Care and Rehabilitation Parameters? A Prospective Evaluation. Int J Phys Med Rehabil S5: 007. doi:10.4172/2329-9096.S5-007

Page 3 of 5

\begin{tabular}{|c|c|}
\hline Previous cardiac operation, n (\%) & $58(11.5)$ \\
\hline Previous stroke, n (\%) & $55(10.9)$ \\
\hline \multicolumn{2}{|l|}{ Previous myocardial infarction } \\
\hline$<6-24$ hours before operation, $n(\%)$ & $22(4.4)$ \\
\hline $1-21$ days before operation, $n(\%)$ & $88(17.4)$ \\
\hline$>22$ days before operation, $n(\%)$ & $65(12.9)$ \\
\hline none, n (\%) & $281(55.6)$ \\
\hline Unknown, n (\%) & $49(9.7)$ \\
\hline Preoperative IABP, n (\%) & $7(1.4)$ \\
\hline Preoperative renal failure, $\mathrm{n}(\%)$ & $15(3.0)$ \\
\hline Urgent operation, n (\%) & $143(28.3)$ \\
\hline \multicolumn{2}{|l|}{ Type of operation } \\
\hline CABG only, $n(\%)$ & $231(45.7)$ \\
\hline CABG and valve, $n(\%)$ & $69(13.7)$ \\
\hline CABG and other, $n(\%)$ & $38(7.5)$ \\
\hline CABG and valve and other, $n(\%)$ & $24(4.8)$ \\
\hline Valve only, n (\%) & $52(10.3)$ \\
\hline Valve and other, $\mathrm{n}(\%)$ & $61(12.1)$ \\
\hline Other, $\mathrm{n}(\%)$ & $30(5.9)$ \\
\hline CPB duration, min & $88.6 \pm 40.6$ \\
\hline Off-pump, n (\%) & $6(1.9)$ \\
\hline Time of operation, min & $190.4 \pm 63.3$ \\
\hline Cross-clamp time, min & $57.6 \pm 27.8$ \\
\hline Intraoperative / early postoperative IABP, $n(\%)$ & $8(1.6)$ \\
\hline \multicolumn{2}{|l|}{ Postoperative complications } \\
\hline Bleeding or tamponade, $\mathrm{n}(\%)$ & $30(5.9)$ \\
\hline Stroke, n (\%) & $8(1.6)$ \\
\hline Renal failure, $\mathrm{n}(\%)$ & $46(9.1)$ \\
\hline 30-day mortality, n (\%) & $17(3.4)$ \\
\hline Length of hospital stay, days, median [IQR] & $9[8-13]$ \\
\hline Length of ICU stay, nights, median [IQR] & $1[1-2]$ \\
\hline Length of IMC stay, days, median [IQR] & $1[0-2]$ \\
\hline \multicolumn{2}{|l|}{ PRN } \\
\hline Daily, points, median [IQR] & $63.8[54.3-77.7]$ \\
\hline Total, points, median [IQR] & $437[311-668]$ \\
\hline First day on ward, points, median [IQR] & $131[86-143]$ \\
\hline \multicolumn{2}{|l|}{ Rehabilitation program } \\
\hline Inpatient, n (\%) & $349(69.1)$ \\
\hline
\end{tabular}

\begin{tabular}{|l|l|}
\hline Outpatient, $\mathrm{n}(\%)$ & $113(22.4)$ \\
\hline None, $\mathrm{n}(\%)$ & $12(2.4)$ \\
\hline Neurologic Rehabilitation, $\mathrm{n}(\%)$ & $4(0.8)$ \\
\hline Unknown, $\mathrm{n}(\%)$ & $10(2.0)$ \\
\hline Data are mean \pm SD unless otherwise specified \\
\hline
\end{tabular}

Table 1: Patient demographics and clinical characteristics

In the univariate analysis, both additive and logistic EuroSCORE were significantly associated with prolonged ICU LOS ( $\mathrm{p}<0.001$ for both correlations, Figure 1), prolonged hospital LOS ( $\mathrm{p}<0.001$ for both correlations, Figure 1), increase in daily nursing effort ( $\mathrm{p}<0.001$ for both correlations, Figure 2), and the type of rehabilitation (inpatient versus outpatient; $\mathrm{p}<0.001$ for both correlations).

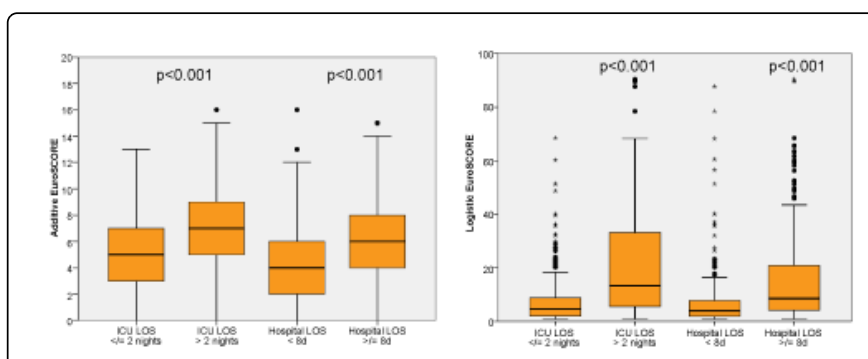

Figure 1: Additive (a) and logistic (b) EuroSCORE correlated to ICU LOS and hospital LOS

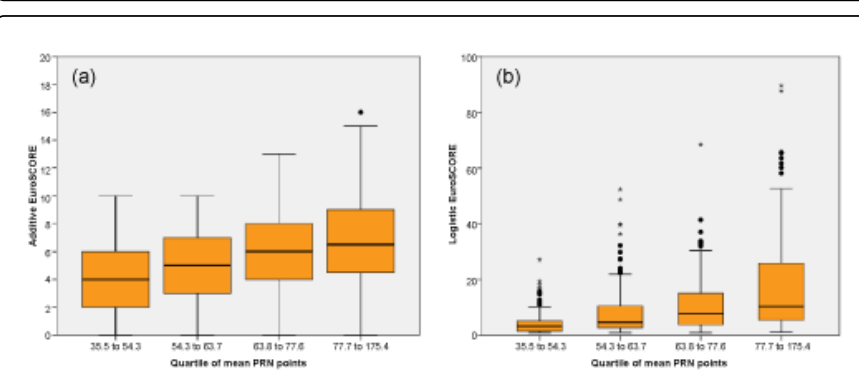

Figure 2: Additive (a) and logistic (b) EuroSCORE correlated to postoperative nursing workload measured by PRN points

Longer $\mathrm{CPB}$ duration $(\mathrm{p}<0.001)$, status of urgent operation $(\mathrm{p}<0.001)$, lower LVEF $(\mathrm{p}=0.015)$ and both higher additive EuroSCORE $(p<0.001)$ and higher logistic EuroSCORE $(p=0.003)$ were independently associated with prolonged ICU LOS. Type of operation, age and BMI were not significantly associated with ICU LOS.

The type of cardiac operation, other than isolated CABG, higher age and both higher additive and higher logistic EuroSCORE were independently associated with prolonged hospital LOS. CPB duration, BMI, status of urgent operation and lower LVEF were not significantly associated with hospital LOS.

In linear regression analysis, mean daily nursing effort was significantly higher in patients with longer CPB duration $(p=0.005)$, higher age $(p=0.016)$ and higher EuroSCORE both additive $(p=0.002)$ 
and logistic $(\mathrm{p}<0.001)$. The type of cardiac operation, BMI, status of urgent operation and lower LVEF were not significantly associated with mean daily nursing effort.

In the multivariate regression analysis, neither additive nor logistic EuroSCORE showed independent association with the type of rehabilitation. The only remaining independent association was detected for the age of the patient: higher age was found to be predictive for inpatient rehabilitation $(\mathrm{p}<0.001$, odds ratio 1.051 with $95 \%$ confidence interval 1.026 to 1.076 ).

\section{Discussion}

Risk stratification models, created for patients undergoing cardiac surgery, are clinically helpful tools in various aspects. Prediction of outcome variables is useful to clinicians in individual patient treatment (e.g. indication for surgery, preoperative counselling) and is, in a more general aspect, the basis for quality monitoring of a hospital's process flow. In addition, risk stratification may be used to estimate needed hospital resources and the amount of overall health care costs for specific treatments [18].

The EuroSCORE model is the most widely evaluated and validated risk stratification system in cardiac surgery $[6,11]]$. Initially developed as an additive scoring system for the prediction of the 30-day mortality, it has been modified later by a logistic regression model [19]. The logistic model shows better individual risk prediction, especially in high-risk patients, whereas the additive EuroSCORE underestimates the risk [20-22].

Besides prediction of 30-day mortality, it has been demonstrated that the EuroSCORE model is also predictive for long-term mortality, specific postoperative complications, long-term health-related quality of life, as well as direct operative costs in patients undergoing cardiac surgery $[3,9,23,24]$. Furthermore, the EuroSCORE model has been used to estimate changes in patients risk profile over time [25].

In the present study we demonstrated that both the additive and the logistic EuroSCORE model independently predict prolonged ICU LOS, prolonged hospital LOS, and postoperative nursing workload. Higher EuroSCORE was associated with inpatient rehabilitation after surgery, but was not independently predictive for the type of cardiac rehabilitation (inpatient versus outpatient).

With respect to the outcome measures ICU LOS and hospital LOS, our study confirms previous findings [5,26]: Ettema and associates validated several prediction models for prolonged ICU stay after cardiac surgery and found that both Parsonnet score and EuroSCORE had superior discrimination compared to other predictive scores [27]. Interestingly, both models widely implemented in clinical practice, were originally derived and validated only for the prediction of mortality after cardiac surgery.

A valid tool for the prediction of ICU LOS und hospital LOS, such as EuroSCORE, is important for clinicians, patients and a hospital's management: Clinicians weigh up the risks and benefits of an indicated operation and perform counselling of patients and relatives in an optimal manner. Patients with predicted lower risk for postoperative complications and estimated short stay on ICU may be planned in the daily operating program before other patients, in order to improve efficiency in the use of operating room and ICU resources [27]. Based on the prediction of ICU LOS and hospital LOS, a hospital's management is enabled to implement strategies for the planning of capacity utilization, the allocation of resources and the estimation of costs.

Nursing workload is a relevant determinant of adequate postoperative care on ICU and regular ward, and a cornerstone in the planning of human resources and the calculation of hospital costs. We were able to demonstrate that EuroSCORE is independently associated with nursing workload on the regular ward. No previous investigations have been focused on this outcome measure. Our findings suggest that patients with higher EuroSCORE do not only have a higher probability for prolonged hospital stay but also for more intense nursing care. For that reason, EuroSCORE seems to be a valid tool for administrative planning of nursing. As a conclusion, surgical units where patients with higher EuroSCORE level are operated need a higher nurse/patient ratio.

Both inpatient and outpatient cardiac rehabilitation programs are evidence-based methods for secondary prophylaxis and reintegration of patients into daily life after cardiac surgery. Information about the anticipated in-hospital postoperative course and further treatment after discharge is essential for patients and their relatives. An early planning of a patient's transfer following hospitalization is wishful for an optimal utilization of a hospital's infrastructure and therefore of hospital costs. To our knowledge, no other study has been focused so far on the association between EuroSCORE and the type of cardiac rehabilitation. Our data demonstrate that EuroSCORE predicts the type of postoperative cardiac rehabilitation in the univariate analysis but not in the multivariate analysis. Out of all tested clinical variables (CPB duration, type of operation, age, BMI, urgency of surgery, LVEF), only higher age was independently associated with inpatient rehabilitation.

Limitations of this trial include the single center design of our investigation. Since inpatient rehabilitation is established only in a few European countries, prediction of the type of rehabilitation is limited to these countries. Furthermore, our institution is a tertiary care university hospital, not having an inpatient rehabilitation center at its disposal. This fact may have led to longer hospital stay. The predictive validity of EuroSCORE for the type of rehabilitation may be limited, because EuroSCORE only weighs up medical variables. However, there are strong social reasons influencing the decision for either inpatient or outpatient rehabilitation. In addition, the availability of appropriate rehabilitation programs as well as the patient's requests may confound our results.

Since the time frame of our study was set before the presentation of the modified EuroSCORE II, we were not able to implement this new version of the predictive model in our study [10]. Further studies are needed to evaluate predictive validity of the new EuroSCORE II regarding other outcome parameters than operative mortality.

A close collaboration between centers for cardiac surgery and rehabilitation facilities is essential for patients, in order to be integrated into a rehabilitation program according to their physical abilities and for early reintegration into daily life.

In conclusion, our prospective observational study confirms that both additive and logistic EuroSCORE are predictive for ICU LOS and hospital LOS after cardiac surgery. We were able to demonstrate the very new aspect that additive and logistic EuroSCORE are also predictive for nursing workload after surgery and for the appropriate type of rehabilitation. EuroSCORE is a widely implemented model for preoperative risk prediction of mortality in cardiac surgery. Therefore, our findings support the use of the EuroSCORE model to detect 
Citation: Goeber V, Faeh U, Keller D, Saner H, Carrel TP, et al. (2014) Does EuroSCORE Predict intensity of Postoperative Care and Rehabilitation Parameters? A Prospective Evaluation. Int J Phys Med Rehabil S5: 007. doi:10.4172/2329-9096.S5-007

Page 5 of 5

patients with elevated risk for prolonged ICU stay and prolonged hospital stay as well as to identify patients who need more intense postoperative nursing care. These latest scientific findings may contribute to optimize the management of hospital bed capacity as well as a systematic planning of ICU resources, postoperative nursing care and cardiac rehabilitation.

\section{Acknowledgements}

The authors wish to thank Brigitta Gahl for excellent statistical support and Sara Baumberger for her assistance in correcting the manuscript's language.

\section{References}

1. Song HK, Diggs BS, Slater MS, Guyton SW, Ungerleider RM, et al. (2009) Improved quality and cost-effectiveness of coronary artery bypass grafting in the United States from 1988 to 2005. J Thorac Cardiovasc Surg 137: 65-69.

2. Pinna Pintor P, Bobbio M, Colangelo S, Veglia F, Marras R, et al. (2003) Can EuroSCORE predict direct costs of cardiac surgery? Eur J Cardiothorac Surg 23: 595-598.

3. Sokolovic E, Schmidlin D, Schmid ER, Turina M, Ruef C, et al. (2002) Determinants of costs and resource utilization associated with open heart surgery. Eur Heart J 23: 574-578.

4. Nilsson J, Algotsson L, Höglund P, Lührs C, Brandt J (2004) EuroSCORE predicts intensive care unit stay and costs of open heart surgery. Ann Thorac Surg 78: 1528-1534.

5. Nashef SAM, Roques F, Michel P, Gauducheau E, Lemeshow S, et al. (1999) European system for cardiac operative risk evaluation (EuroSCORE). Eur J Cardiothorac Surg 16: 9-13.

6. Roques F, Nashef SA, Michel P, Gauducheau E, de Vincentiis C, et al. (1999) Risk factors and outcome in European cardiac surgery: analysis of the EuroSCORE multinational database of 19030 patients. Eur J Cardiothorac Surg 15: 816-822.

7. Roques F, Nashef SA, Michel P, Pinna Pintor P, David M, et al. (2000) Does EuroSCORE work in individual European countries? Eur J Cardiothorac Surg 18: 27-30.

8. Nashef SA, Roques F, Hammill BG, Peterson ED, Michel P, et al. (2002) Validation of European System for Cardiac Operative Risk Evaluation (EuroSCORE) in North American cardiac surgery. Eur J Cardiothorac Surg 22: 101-105.

9. Pinna Pintor P, Bobbio M, Colangelo S, Veglia F, Marras R, et al. (2003) Can EuroSCORE predict direct costs of cardiac surgery? Eur J Cardiothorac Surg 23: 595-598.

10. Nashef SA, Roques F, Sharples LD, Nilsson J, Smith C, et al. (2012) EuroSCORE II. Eur J Cardiothorac Surg 41: 734-744.

11. Gogbashian A1, Sedrakyan A, Treasure T (2004) EuroSCORE: a systematic review of international performance. Eur J Cardiothorac Surg 25: 695-700.

12. Chagnon M, Audette L, Lebrun L, Tilquin C (1978) A patient classification system by levels of nursing care requirements. Nurs Res 27: 107-113.
13. Cockerill R, Pallas LO, Bolley H, Pink G (1993) Measuring nursing workload for case costing. Nurs Econ 11: 342-349.

14. Chagnon M, Audette LM, Lebrun L, Tilquin C (1978) Validation of a patient classification through evaluation of the nursing staff degree of occupation. Med Care 16: 465-475.

15. O'Brien-Pallas L, Leatt P, Deber R, Till J (1989) A comparison of workload estimates using three methods of patient classification. Can J Nurs Admin 2: 16-23.

16. Hall LM1, Pink L, Lalonde M, Murphy GT, O'Brien-Pallas L, et al. (2006) Decision making for nurse staffing: Canadian perspectives. Policy Polit Nurs Pract 7: 261-269.

17. http://www.sts.org/sites/default/files/documents/pdf/ndb2010/ Report_OV_General_5-37.pdf

18. Shahian DM, Blackstone EH, Edwards FH, Grover FL, Grunkemeier GL, et al. (2004) Cardiac surgery risk models: a position article. Ann Thorac Surg 78: 1868-1877.

19. Roques F, Michel P, Goldstone AR, Nashef SA (2003) The logistic EuroSCORE. Eur Heart J 24: 881-882.

20. Michel P, Roques F, Nashef SA; EuroSCORE Project Group (2003) Logistic or additive EuroSCORE for high-risk patients? Eur J Cardiothorac Surg 23: 684-687.

21. Jin R, Grunkemeier GL; Providence Health System Cardiovascular Study Group (2005) Additive vs. logistic risk models for cardiac surgery mortality. Eur J Cardiothorac Surg 28: 240-243.

22. Zingone B, Pappalardo A, Dreas L (2004) Logistic versus additive EuroSCORE. A comparative assessment of the two models in an independent population sample. Eur J Cardiothorac Surg 26: 1134-1140.

23. Toumpoulis IK, Anagnostopoulos CE, Swistel DG, DeRose JJ Jr (2005) Does EuroSCORE predict length of stay and specific postoperative complications after cardiac surgery? Eur J Cardiothorac Surg 27: 128-133.

24. Loponen P, Luther M, Nissinen J, Wistbacka JO, Biancari F, et al. (2008) EuroSCORE predicts health-related quality of life after coronary artery bypass grafting. Interact Cardiovasc Thorac Surg 7: 564-568.

25. Ohata T, Kaneko M, Kuratani T, Ueda H, Shimamura K (2005) Using the EuroSCORE to assess changes in the risk profiles of the patients undergoing coronary artery bypass grafting before and after the introduction of less invasive coronary surgery. Ann Thorac Surg 80: 131-135.

26. Messaoudi N, De Cocker J, Stockman BA, Bossaert LL, Rodrigus IE (2009) Is EuroSCORE useful in the prediction of extended intensive care unit stay after cardiac surgery? Eur J Cardiothorac Surg 36: 35-39.

27. Ettema RG, Peelen LM, Schuurmans MJ, Nierich AP, Kalkman CJ, et al. (2010) Prediction models for prolonged intensive care unit stay after cardiac surgery: systematic review and validation study. Circulation 122: $682-689,7$ p following p 689. 